\title{
A case of pseudomyxoma peritonei successfully treated with trifluridine/tipiracil (TAS-102) and bevacizumab after palliative debulking surgery
}

\author{
Satoshi Hirano ${ }^{1}$, Yoshimasa Gohda ${ }^{2}$, Hideki Miyazaki ${ }^{3}$, Nami Hayama ${ }^{4}$, Shinichiro Shimizu ${ }^{5}$ Toru Igari $^{3}$, \\ Hideaki Yano ${ }^{2}$ \\ ${ }^{1}$ Department of Medical Oncology, Funabashi Municipal Medical Center, Chiba, Japan; ${ }^{2}$ Department of Surgery, National Center for Global \\ Health and Medicine, Tokyo, Japan; ${ }^{3}$ Pathology Division of Clinical Laboratory, National Center for Global Health and Medicine, Tokyo, Japan; \\ ${ }^{4}$ Department of Respiratory Medicine, Funabashi Municipal Medical Center, Chiba, Japan; ${ }^{5}$ Department of Pathology, Funabashi Municipal Medical \\ Center, Chiba, Japan \\ Correspondence to: Satoshi Hirano. Department of Medical Oncology, Funabashi Municipal Medical Center, 1-21-1 Kanasugi, Funabashi City, Chiba \\ 273-8588, Japan. Email: shirano@mmc.funabashi.chiba.jp.
}

\begin{abstract}
Pseudomyxoma peritonei is a rare disease with a reported prevalence of about 1-3 per million people annually. Cytoreductive surgery and perioperative hyperthermic intraperitoneal chemotherapy are considered as treatment options improving disease control or long-term survival. However, for patients with incomplete cytoreduction or debulking surgery, outcomes are significantly poorer compared with patients who have obtained complete or optimal cytoreduction. In cases of high-grade pseudomyxoma peritonei that are considered inoperable and/or unresectable, combination chemotherapy regimen with a neo-angiogenesis inhibitor such as bevacizumab is recommended. In this report, a 62-year-old Japanese man presented with abdominal distention. Examination of ascites demonstrated a jelly-like consistency and peritoneal pseudomyxoma was suspected. To relieve progressive symptoms, palliative debulking surgery with total colectomy was performed. Postoperative pathology confirmed high-grade appendiceal mucinous neoplasm with high-grade pseudomyxoma peritonei. In our case, combination chemotherapy with trifluridine/tipiracil (TAS-102) and bevacizumab was initiated after palliative debulking surgery. As a result, carcinoembryonic antigen level was kept stable and the volume of ascites remained almost the same as at the beginning of treatment for more than 2 years. In conclusion, combination chemotherapy comprising TAS-102 and bevacizumab in patients with palliative debulking could be a useful option for patients with high-grade mucinous appendiceal neoplasm and high-grade pseudomyxoma peritonei.
\end{abstract}

Keywords: Pseudomyxoma peritonei (PMP); trifluridine/tipiracil (TAS-102); bevacizumab

Submitted Jan 02, 2021. Accepted for publication Feb 15, 2021.

doi: $10.21037 /$ cco-21-2

View this article at: http://dx.doi.org/10.21037/cco-21-2

\section{Introduction}

Pseudomyxoma peritonei (PMP) is a rare disease with a reported prevalence of about $1-3$ per million people annually (1). Cytoreductive surgery (CRS) and perioperative hyperthermic intraperitoneal chemotherapy (HIPEC) are considered as treatment options allowing improved disease control and better long-term survival $(2,3)$. However, for patients with incomplete cytoreduction or debulking surgery, the outcomes are significantly poorer compared with those in patients who have obtained complete or

^ ORCID: 0000-0001-5392-8337. 
optimal cytoreduction (3). In cases where high-grade PMP is considered inoperable and/or unresectable, a combination chemotherapy regimen including a neo-angiogenesis inhibitor such as bevacizumab is recommended (2). Our case suggests that combination chemotherapy with trifluridine/tipiracil (TAS-102) and bevacizumab after palliative debulking surgery may be beneficial for patients with high-grade appendiceal mucinous neoplasm (HAMN) accompanied by PMP.

We present the following case in accordance with the CARE reporting checklist (available at http://dx.doi. org/10.21037/cco-21-2).

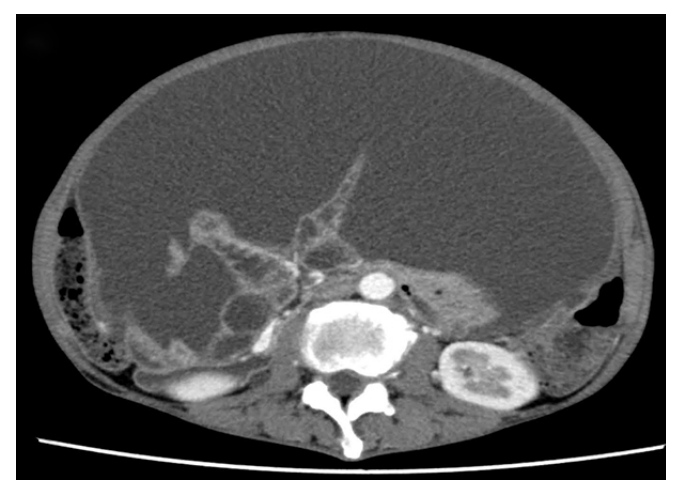

Figure 1 Contrast-enhanced abdominal CT. Before palliative debulking surgery, the abdominal wall is severely distended due to accumulation of slightly hyperdense mucinous ascites and the small intestine is severely compressed. CT, computed tomography.

\section{Case presentation}

A 62-year-old Japanese man presented to a local hospital with abdominal distention. His past medical history was not remarkable. Massive ascites was demonstrated on abdominal and pelvic computed tomography. Adenocarcinoma with unknown primary was diagnosed from cytology of ascites. Eight courses of chemotherapy with carboplatin and paclitaxel, followed by eight courses of gemcitabine were performed at the hospital. However, his general condition deteriorated and he was admitted to Funabashi Municipal Medical Center.

Despite chemotherapy with gemcitabine, ascites continued to increase (Figure 1). Examination of ascites demonstrated a jelly-like consistency and peritoneal pseudomyxoma was suspected. To relieve progressive symptoms, palliative debulking surgery (CCR3) with total colectomy was performed in the National Center for Global Health and Medicine. Postoperative pathology confirmed HAMN with high-grade PMP (Figure 2). KRAS p.Gly12Asp missense mutation was demonstrated, but no microsatellite instability was evident.

Postoperatively, chemotherapies for colon cancer were repeated for the residual lesion in Funabashi Municipal Medical Center. The patient received FOLFOX + bevacizumab, FOLFIRI + ramucirumab, regorafenib, and gemcitabine, none of which showed sufficient efficacy. As a result, his levels of carcinoembryonic antigen (CEA) gradually elevated. Finally, the patient received treatment with bevacizumab $(7.5 \mathrm{mg} / \mathrm{kg})$ plus TAS-102
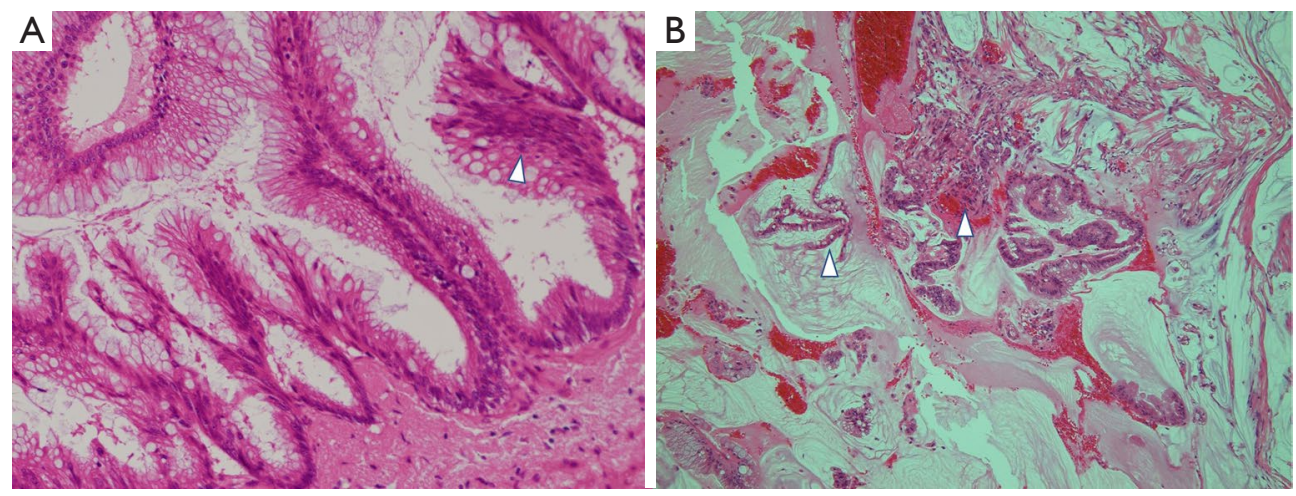

Figure 2 Histopathology of the primary lesion (A, HE stain, 200x) and disseminated lesion (B, HE stain, 100x). (A) Papillary or dendritic proliferation with swelling, enlargement, and stratification of the nucleus (arrow), equivalent to high-grade appendiceal mucinous neoplasm. (B) Tumor epithelium is demonstrated at the margins of mucus nodules (arrow). Abundant fragments of neoplastic mucinous epithelium are seen floating in the mucus (arrow). 


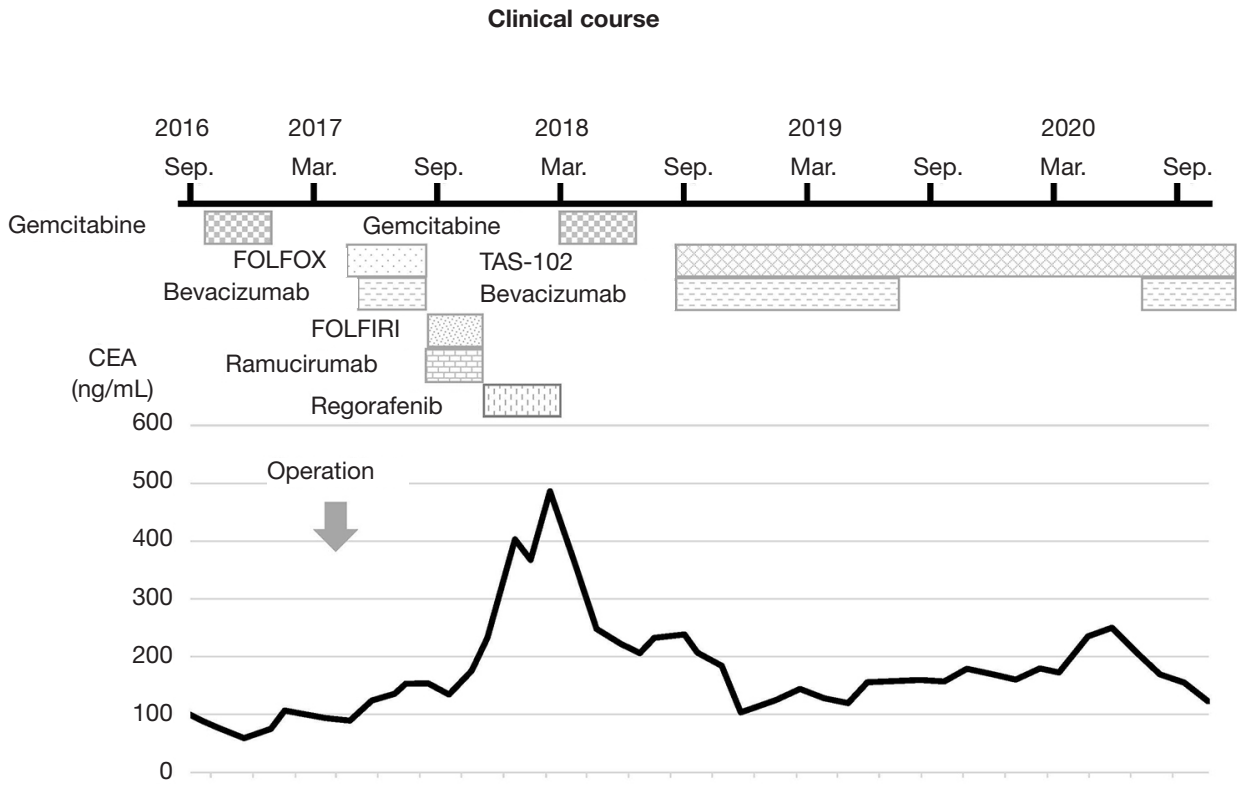

FOLFOX, 5-fluorouracil + oxaliplatin; FOLFIRI, 5-fluorouracil + irinotecan;

TAS-102, trifluridine, tipiracil hydrochloride

Figure 3 Clinical course. Chemotherapy regimen and dynamics of tumor marker CEA. The tumor marker seems stable during bevacizumab and trifluridine/tipiracil (TAS-102) treatment. CEA, carcinoembryonic antigen.

( $35 \mathrm{mg} / \mathrm{m}^{2}$ twice daily on days $1-5$ and $8-12$ ), in $28-$ or $35-$ day cycles. Due to worsening of proteinuria, bevacizumab was interrupted for about 1 year, but CEA level was kept stable for more than 2 years and the volume of ascites remained almost the same as that at the beginning of treatment (Figures 3,4). Other than repeated ileus for short periods due to postoperative adhesions, the main adverse events were numbness in the hands and feet, fatigue, loss of appetite and neutropenia.

All procedures performed in studies involving human participants were in accordance with the ethical standards of the institutional and/or national research committee(s) and with the Helsinki Declaration (as revised in 2013). Written informed consent was obtained from the patient.

\section{Discussion}

PMP is a rare peritoneal malignancy characterized by diffuse, progressive, mucinous ascites. PMP has recently been mostly considered as originating from perforated epithelial tumor of the appendix, but is a biologically heterogeneous disease $(2,3)$. Generally, the Peritoneal Surface Oncology Group International (PSOGI) consensus classification has been accepted globally. The new concept of HAMN was proposed for lesions without infiltrative invasion, but showing high-grade cytological atypia in 2016 (4). The histopathological characteristics of HAMN includes, at least focally, loss of polarity with full-thickness nuclear stratification, nuclei that are enlarged, markedly hyperchromatic or vesicular, prominent nucleoli, numerous or atypical mitotic figures, or cribriform growth (2). Some of these characteristics were demonstrated in our case (Figure 2A). Three categories of PMP have been recognized: low grade; high grade; and high grade with signet ring cells. In this classification, high-grade mucinous carcinoma peritonei is considered synonymous with peritoneal mucinous carcinomatosis. In our case, HAMN with high-grade PMP was confirmed from postoperative pathology.

CRS and perioperative HIPEC are thought to be important treatment options for improving disease control and long-term survival $(2,3)$. However, for patients with incomplete cytoreduction analogous to debulking surgery (CCR2 or CCR3) in whom gross residual disease is present, outcomes are significantly poorer even when stratified by histopathological subtype on multivariate analysis (3). 

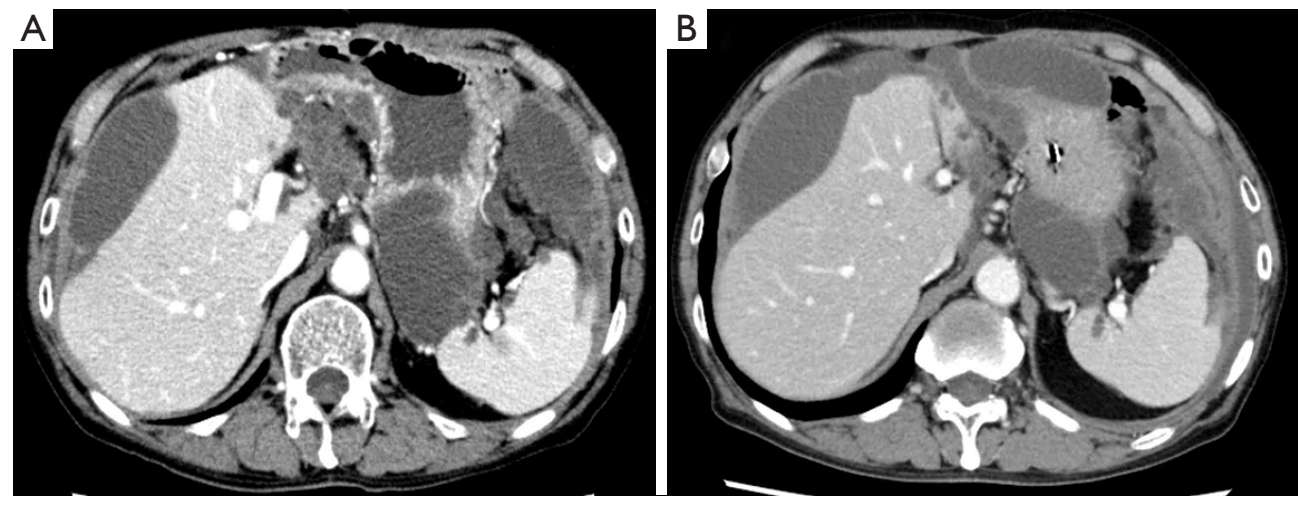

Figure 4 Contrast-enhanced abdominal CT. The amount of loculated residual mucinous ascites is almost the same before (A) and 2 years after starting chemotherapy with bevacizumab and trifluridine/tipiracil (TAS-102) (B). CT, computed tomography.

For patients with recurrent, or unresectable, PMP treated with palliative systemic chemotherapy, progression-free survival (PFS) ranged from 6 to 8 months, and overall survival (OS) ranged from 26 to 61 months (2). For patients with recurrent or unresectable PMP, especially those with higher-grade appendiceal epithelial neoplasms, bevacizumab (a monoclonal antibody blocking the vascular endothelial growth factor ligand) in combination with chemotherapy showed improvements in PFS and OS. Most regimens in their study comprised 5-fluorouracil-based therapies, such as 5-fluorouracil/capecitabine monotherapy, FOLFOX/ CapeOX, or FOLFIRI/CapeIRI (5). On the other hand, some PMP experts have suggested that a combination of fluoropyrimidine and an alkylating agent like oxaliplatin together with a neo-angiogenesis inhibitor like bevacizumab should be considered (2). In our case, several chemotherapy regimens for patients with colorectal cancer (FOLFOX plus bevacizumab, FOLFIRI plus ramucirumab, regorafenib) were administered, none of which showed sufficient efficacy.

Sun et al. showed that a combination of bevacizumab and capecitabine following FOLFOX 4 chemotherapy for progressive disease led to long-term stabilization of the disease. They also showed that the level of the tumor marker carbohydrate antigen 19-9 offered the most useful index for follow-up of systemic chemotherapy together with radiological evaluation (6).

TAS-102 is an oral nucleoside antitumor agent containing trifluridine and tipiracil. Trifluridine is the antitumor component of TAS-102, whereas tipiracil prevents degradation of trifluridine as a thymidine phosphorylase inhibitor (7). TAS-102 has shown significant OS benefits compared with placebo in patients with chemorefractory metastatic colorectal cancer $(8,9)$. Moreover, TAS-102 plus bevacizumab, as compared with TAS-102 monotherapy, has shown significant improvements in progression-free survival for patients with chemorefractory metastatic colorectal cancer (10).

We selected a regimen of TAS-102 plus bevacizumab as the seventh-line treatment for this patient. In our case, changes in CEA level seemed more useful than radiological examinations, because the residual lesions comprised mucinous ascites with no obvious target mass or nodular lesions, similar to most other PMP patients. During the period of bevacizumab interruption as a key drug for the treatment of PMP due to exacerbation of proteinuria, CEA seemed to be temporarily elevated. However, after reinitiation, tumor markers remained stable. Although no significant decrease in ascites was identified, CEA levels remained broadly stable for more than 2 years. Considering the necessity for continuing chemotherapy for long periods, treatment with TAS-102 and bevacizumab could represent an important treatment option given their mild toxicity profiles.

The limitation of this report is that this knowledge was derived only from one patient as this disease is quite rare. Further reports of similar cases might provide more robust evidence.

In conclusion, combination chemotherapy comprising TAS-102 and bevacizumab in patients with palliative debulking could be a useful option for patients with highgrade mucinous appendiceal neoplasm and high-grade PMP.

\section{Acknowledgments}

Funding: None. 


\section{Footnote}

Reporting Checklist: The authors have completed the CARE reporting checklist. Available at http://dx.doi.org/10.21037/ cco-21-2

Peer Review File: Available at http://dx.doi.org/10.21037/ cco-21-2

Conflicts of Interest: All authors have completed the ICMJE uniform disclosure form (available at http://dx.doi. org/10.21037/cco-21-2). The authors have no conflicts of interest to declare.

Ethical Statement: The authors are accountable for all aspects of the work in ensuring that questions related to the accuracy or integrity of any part of the work are appropriately investigated and resolved. All procedures performed in studies involving human participants were in accordance with the ethical standards of the institutional and/or national research committee(s) and with the Helsinki Declaration (as revised in 2013). Written informed consent was obtained from the patient.

Open Access Statement: This is an Open Access article distributed in accordance with the Creative Commons Attribution-NonCommercial-NoDerivs 4.0 International License (CC BY-NC-ND 4.0), which permits the noncommercial replication and distribution of the article with the strict proviso that no changes or edits are made and the original work is properly cited (including links to both the formal publication through the relevant DOI and the license). See: https://creativecommons.org/licenses/by-nc-nd/4.0/.

\section{References}

1. Rizvi SA, Syed W, Shergill R. Approach to pseudomyxoma peritonei. World J Gastrointest Surg 2018;10:49-56.

2. Govaerts K, Lurvink RJ, De Hingh IHJT, et al.

Cite this article as: Hirano S, Gohda Y, Miyazaki H, Hayama N, Shimizu S, Igari T, Yano H. A case of pseudomyxoma peritonei successfully treated with trifluridine/tipiracil (TAS102) and bevacizumab after palliative debulking surgery. Chin Clin Oncol 2021;10(3):29. doi: 10.21037/cco-21-2
Appendiceal tumours and pseudomyxoma peritonei: Literature review with PSOGI/EURACAN clinical practice guidelines for diagnosis and treatment. Eur J Surg Oncol 2021;47:11-35.

3. Chua TC, Moran BJ, Sugarbaker PH, et al. Early- and long-term outcome data of patients with pseudomyxoma peritonei from appendiceal origin treated by a strategy of cytoreductive surgery and hyperthermic intraperitoneal chemotherapy. J Clin Oncol 2012;30:2449-56.

4. Carr NJ, Cecil TD, Mohamed F, et al. A Consensus for Classification and Pathologic Reporting of Pseudomyxoma Peritonei and Associated Appendiceal Neoplasia: The Results of the Peritoneal Surface Oncology Group International (PSOGI) Modified Delphi Process. Am J Surg Pathol 2016;40:14-26.

5. Choe JH, Overman MJ, Fournier KF, et al. Improved Survival with Anti-VEGF Therapy in the Treatment of Unresectable Appendiceal Epithelial Neoplasms. Ann Surg Oncol 2015;22:2578-84.

6. Sun WL, Hutarew G, Gradl J, et al. Successful antiangiogenic combination therapy for pseudomyxoma peritonei with bevacizumab and capecitabine. Cancer Biol Ther 2009;8:1459-62.

7. Tanaka N, Sakamoto K, Okabe H, et al. Repeated oral dosing of TAS-102 confers high trifluridine incorporation into DNA and sustained antitumor activity in mouse models. Oncol Rep 2014;32:2319-26.

8. Yoshino T, Mizunuma N, Yamazaki K, et al. TAS-102 monotherapy for pretreated metastatic colorectal cancer: a double-blind, randomised, placebo-controlled phase 2 trial. Lancet Oncol 2012;13:993-1001.

9. Mayer RJ, Van Cutsem E, Falcone A, et al. Randomized trial of TAS-102 for refractory metastatic colorectal cancer. N Engl J Med 2015;372:1909-19.

10. Pfeiffer P, Yilmaz M, Möller S, et al. TAS-102 with or without bevacizumab in patients with chemorefractory metastatic colorectal cancer: an investigator-initiated, open-label, randomised, phase 2 trial. Lancet Oncol 2020;21:412-20. 